\title{
Resistance of soybean genotypes to white mold in distinct agroecosystems
}

\author{
Gabriela Carolina Guimarães Andrade ${ }^{1}$, Renato Carrer Filho ${ }^{1}$, Marcos Gomes da Cunha ${ }^{1}$ \\ ${ }^{1}$ Universidade Federal de Goiás - UFG, Campus Goiânia, GO, Brasil. E-mail: gabriela.andrade@ bayer.com, \\ carrerfilho@hotmail.com,mgcunha1@gmail.com,
}

Recebido: 11/06/2017; Aceito: 03/02/2018

\begin{abstract}
White mold (Sclerotinia sclerotiorum) is one of the most important fungal diseases that affect soybean, primarily due to the production of resistant structures called sclerotia. The disease is difficult to control, and sources of genetic resistance are poorly understood. As such, the present study aimed to assess the resistance of soybean genotypes to white mold in two agroecosystems in the Brazil (Barreiras-BA, and Jataí-GO) and analyze the relationship between the disease incidence and the phenotypic characteristics of the genotypes, as well as determine the lodging index, crop cycle and yield. A total of 165 and 33 genotypes were assessed in the regions of Barreiras and Jataí, respectively. To verify the effect of the area, 37 genotypes were planted in both regions. The area effect was assessed for the study variables, and the correlation was significant between the disease and lodging, cycle and production. Considering resistance, area and yield, genotypes ANTA82, 2011L003, 2011L005 exhibited the highest yield and resistance to white mold.
\end{abstract}

Palavras-chave: Glycine max; Sclerotinia sclerotiorum; plant improvement; genetic control

\section{Resistência de genótipos de soja ao mofo-branco em distintos agroecossistemas}

\section{RESUMO}

O mofo-branco (Sclerotinia sclerotiorum) destaca-se como uma das principais doenças fúngicas que acometem a soja, devido principalmente à produção, por parte do patógeno, de estruturas de resistência chamadas escleródios. A doença é de difícil controle e fontes de resistência genética são pouco conhecidas. Dessa maneira, o objetivo do presente trabalho foi avaliar a resistência de genótipos de soja ao mofo branco em dois agroecossistemas (Barreiras/BA e Jataí/GO) e analisar a relação da incidência da doença com características fenotípicas dos genótipos, bem como determinar o índice de acamamento, ciclo da cultura e produtividade. Na região de Barreiras foram avaliados 165 genótipos e na região de Jataí, 63 genótipos. Para verificar efeito de local, 37 genótipos foram plantados em ambas as regiões. O efeito local foi verificado para as variáveis estudadas e a correlação foi significativa entre a incidência da doença e o acamamento, o ciclo e a produção. Levando-se em consideração a resistência, local e produtividade, os genótipos ANTA82, 2011L003, 2011L005 se destacaram dos demais com maior produtividade e resistência ao mofo branco

Key words: Glycine max, Sclerotinia sclerotiorum, melhoramento de plantas. 


\section{Introduction}

Soybean [Glycine $\max$ (L.) Merr.], currently the most important crop in the country is grown in all the geographic regions of Brazil, the second largest producer and leading exporter of soybean worldwide (CONAB, 2015). Among the main problems that can take to decreased productivity, diseases are ones of the primary causes of yield loss, highlighting the fungus Sclerotinia sclerotiorum (Lib.) de Bary, the causative agent of white mold, also known as soybean stem white rot (JULIATTI et al., 2013b). This aggressive, polyphagous, devastating and difficult-to-control species is recognized as one of the most critical crop pathosystems (BOLTON et al., 2006; ZHAO et al., 2015), with damage affecting more than $60 \%$ of Central Brazil (CUNHA et al., 2010; WRATHER et al., 2010).

The disease is difficult to control, and sources of genetic resistance are little known. Concerning the control of plant diseases, the use of known resistant varieties has produced better, more economical results that have less effect on the environment (WRATHER et al., 2010). Complete resistance to white mold has yet to be reported in soybean crops (KURLE et al., 2001), and the disease seems to be governed by quantitative traits (QTL), suggesting a multiple-locus model (ZHAO et al., 2015).

Partial resistance to $S$. sclerotiorum in soybean cultivars has been identified in field assessments, but current resistance sources of commercial cultivars are limited and do not prevent significant crop yield loss (WEGULO et al., 1998; YANG et al., 1999; KIM; DIERS, 2000). For more efficient control, the use of genotypes that exhibit partial resistance deserves attention. Only partial resistance associated with escape mechanisms or physiological resistance to $S$. sclerotiorum has been widely used (HOFFMAN et al., 2002).

Little is known in Brazil about the partial resistance of soybean varieties to white mold, which limits the development of germplasm with this type of resistance. Since partial resistance may promote economically viable control of the disease, it should be the object of soybean breeding programs. However, studies on soybean resistance to white mold are scarce, prompting the development of breeding strategies to assess the effects of $S$. sclerotiorum in future cultivars (KIM; DIERS, 2000; GARCIA et al., 2015). Given the importance of soybean in Brazil and concern about compromised production caused by increasing yield losses, assessing the resistance of genotypes to $S$. sclerotiorum is a key component in the integrated management of the disease. As such, this study aimed to assess the resistance of soybean genotypes to white mold and study the relationship between the disease incidence and the phenotypic characteristics of the genotypes.

\section{Material and Methods}

The two experiments were conducted in an area naturally infested by Sclerotinia sclerotiorum, in the municipalities of Barreiras in Bahia (BA) state and Jataí, Goias (GO) state, Brazil, during the 2011/12 growth season. In both regions, planting involved a conventional system, using a four-row seeder in the experimental plots, without chemical spraying to control white mold. Population density ranged from 10 to 18 plants per linear meter, in line with cultivar recommendations for each region, according to the breeding company.

In the municipality of Barreiras, BA, planting occurred on November 14 and 165 genotypes were used, 17 commercial cultivars and 148 breeding lines in the first and second year of value for cultivation and use (VCU), from maturity groups between 7.5 and 9.1. In Jataí, GO, planting was carried out on October 12, and 63 soybean genotypes were used, 13 commercial cultivars and 50 strains in the first and second year of VCU, from maturity groups between 6.8 and 8.2. A total of 37 genotypes were tested in each area, 30 strains in the first and second year of VCU and seven commercial cultivars, from maturity groups ranging from 7.0 to 8.2. The experimental design was conducted in random blocks with three repetitions, 165 treatments in Barreiras-BA and 63 in Jataí-GO. The experimental plots consisted of four 6-meter long rows spaced $0.5 \mathrm{~m}$ apart, the study area is the two central rows, eliminating $0.5 \mathrm{~m}$ at both ends.

The disease was assessed 90 days after planting (DAP) when the materials were between phases R4 and R6 (FEHR et al., 1971). Disease incidence was assessed in the plants from the two central rows of the plot, using a 9-point scale, where $1=0 \%$ of infected plants; $2=1$ to $10 \% ; 3=11$ to $20 \% ; 4=21$ to $35 \% ; 5=36$ to $50 \% ; 6$ $=51$ to $65 \% ; 7=66$ to $75 \% ; 8=76$ to $85 \% ; 9=>85 \%$. The agronomic characteristics lodging, yield and cultivar cycle were also assessed in each area. For the first characteristic, a 9-point scale adapted by Bernard et al. (1965) was used, as follows: $1=$ erect plants and $9=$ extremely lodged plants. The yield was obtained by harvesting the two central rows of the plots. The yield was measured, and bean moisture content determined using a Gehaka G600i portable moisture meter. The cycle of each cultivar was quantified as the number of days between planting and physiological maturity, that is, phenological stage R7.

The incidence rate, lodging, yield and cycle data were submitted to analysis of variance and, when significant, the means were cluster using the Scott-Knott 
test $(P<0.05)$. Pearson's correlation coefficient $(P<0.05)$ was determined by disease incidence and the lodging index, genotype cycles and yield $\left(\mathrm{kg} \mathrm{ha}^{-1}\right)$. The Sisvar 5.3 system (FERREIRA, 2011) was used for analysis.

\section{Results and Discussion}

Assessment of disease incidence in Barreiras/BA showed the formation of 3 response to white mold groups. Group 1 exhibited high resistance, with incidence scores between 1.11 and 3.33. This group was composed of 212 genotypes, five commercial cultivars and 16 strains. Group 2 displayed intermediate resistance, with an incidence ranging from 3.67 to 5.40 and consisting of 49 genotypes, seven commercial cultivars and 42 strains. Group 3, the most susceptible, contained 95 genotypes, five cultivars and 90 strains (Table 1).

The cultivars ANTA82, P98Y12, M7639RR, NA7337RR and P98Y30, showed the lowest disease incidence, followed by 2011L003 and 2011L84, and 14 more strains. The cultivars ANTA82 and NA7337RR were considered a super-early cycle for the region, while P98Y12, 2011L84, 2011L003 and M7639RR exhibited an early cycle (Table 1). As such, in both cases, the assessment of disease incidence the genotypes in Barreiras/BA, these may have been favored by possible disease escape due to early maturation. According to Yang et al. (1999), the incidence of $S$. sclerotiorum in soybean cultivars is related to the maturation groups. According to these authors, longcycle cultivars are more susceptible, due to the more extended flowering period, which causes higher predisposition to infection by ascospores. Cultivar P98Y30, which exhibited an average cycle in the region, is promising concerning resistance to white mold since it showed an incidence rate of 1.93 .

The culture cycle is a factor that interferes with the final severity of the disease. The longer the culture in the field, the higher the chance of disease occurring. The lodging index is another a variable that must be considered, since resistant to bedding cultivars with upright plants, good air circulation and rapid drying within the canopy of the crop are factors that can significantly reduce the intensity of white mold in soybean. About lodging index, five groups were formed. The first group contained 61 genotypes with a lodging index between 1.00 and 1.80, considered the most erect, with ten cultivars and 51 strains. In the second group, the lodging ranged from 2.00 to 2.33 and consisted of 35 genotypes, three cultivars and 32 strains. The third group, with lodging between 2.67 and 3.67, was composed of 29 genotypes, three cultivars and 26 strains. The fourth and fifth groups, with intermediateto-high lodging indices, were represented by 39 strains
(Table 1). Of the 17 commercial cultivars tested, ten belonged to the first group, namely ANTA82, P98Y12, NA7337RR, NA7255RR and P98Y51, followed by P98Y30, M9144RR, P98Y11, TMG-132RR and P98Y70. The lodging index is a variable that should be considered, given that plants of lodging-resistant cultivars are the most erect, allowing good air circulation and rapid elimination of moisture in the canopy of the crop. These characteristics are factors that may significantly reduce the incidence of white mold in soybean (BOLAND; HALL, 1987; KIM et al., 1999; JULIATTI et al., 2013a).

About yield, two groups were formed. The first group, with 73 genotypes, consisted of 66 strains and seven cultivars, with a yield ranging from $6,147 \mathrm{~kg} \mathrm{ha}^{-1}$ to $4,736 \mathrm{~kg} \mathrm{ha}^{-1}$. The second group, with a yield between 4,701 $\mathrm{kg} \mathrm{ha}^{-1}$ and 2,247 $\mathrm{kg} \mathrm{ha}^{-1}$, was composed of 92 genotypes, 82 strains and ten cultivars (Table 1). The most productive of the commercial cultivars was P98Y51, which did not differ significantly from P98Y70, M7639RR, TMG-132RR, M8527RR, P99R03 and M9144RR. The least productive was AS8380RR, which did not differ statistically from P98Y12, P98Y11, NA7255RR, TMG1176RR, M7908RR, M8230RR, NA7337RR, ANTA82 and P98Y30.

In the trial conducted in Jataí and Barreiras, three groups of cultivars were observed based on the incidence rate, considering the 63 genotypes tested (Table 2). The first group, the most resistant, with an incidence rate ranging from 1 to 2.33 , consisted of 12 genotypes, with strains 2011L003, 2010L013 and 2010L011 obtaining the lowest rates, followed by the commercial cultivars NA7337RR and ANTA82, and seven other strains. Group two, considered moderately susceptible, with an incidence rate between 3 and 4.33, was composed of 14 genotypes, two cultivars (POTÊNCIA-RR and VMAXRR), and 12 strains. Group three, the most susceptible, consisted of 37 genotypes, nine cultivars and 28 strains.

Of the strains with a low incidence rate for white mold, 2010L013 and 2010L011 exhibited an early cycle in the region, while 2011L003 displayed a late cycle and the other genotypes an intermediate cycle. Cultivar ANTA82 showed the highest resistance, lowest lodging index and was classified in the super-early maturation group in Barreiras/BA, which may have favored disease escape, but in Jataí/GO this cultivar exhibited an intermediate cycle and moderate resistance to $S$. sclerotiorum (Table 2), which may lead to the conclusion that other factors besides the cycle may have disadvantaged the development of the disease. As the strain 2011L003, since it is a late cycle, thereby favoring the development of the disease, deserves attention in future studies given that it is resistant to white mold in the two regions. 
Table 1. Incidence rate of Sclerotinia sclerotiorum, grain yield, lodging index and the cycle of 165 soybean genotypes assessed in Barreiras, BA, Brazil in the 2011/2012 growing season

\begin{tabular}{|c|c|c|c|c|c|c|}
\hline Genotypes & Denomination & $\begin{array}{l}\text { Incidence } \\
\text { rate }\end{array}$ & $\begin{array}{l}\text { Yield } \\
\mathrm{kg} \mathrm{ha}^{-1}\end{array}$ & Lodging index & Cycle (days) & Classification \\
\hline ANTA82 & Cultivar & $1.11 \mathrm{a}$ & $4,522 \mathrm{~b}$ & $1.00 \mathrm{a}$ & 101.7 & Super-early \\
\hline P98Y12 & " & $1.33 \mathrm{a}$ & $3,878 \mathrm{~b}$ & $1.00 \mathrm{a}$ & 113.0 & Early \\
\hline M7639RR & $"$ & $1.67 \mathrm{a}$ & $5,211 \mathrm{a}$ & $2.00 \mathrm{~b}$ & 107.8 & Super-early \\
\hline NA7337RR & $"$ & $1.67 \mathrm{a}$ & $4,311 \mathrm{~b}$ & $1.00 \mathrm{a}$ & 108.0 & Super-early \\
\hline 2011L003 & Lineage & $1.67 \mathrm{a}$ & $4,756 \mathrm{a}$ & $1.67 \mathrm{a}$ & 110.0 & Early \\
\hline 2011L084 & " & $1.67 \mathrm{a}$ & $4,340 \mathrm{~b}$ & $1.33 \mathrm{a}$ & 119.3 & Early \\
\hline P98Y30 & Cultivar & $1.93 \mathrm{a}$ & $4,644 \mathrm{~b}$ & $1.20 \mathrm{a}$ & 122.0 & Average \\
\hline 2011L005 & Lineage & $2.33 \mathrm{a}$ & $4,635 \mathrm{~b}$ & $1.00 \mathrm{a}$ & 113.0 & Early \\
\hline 2011L006 & " & $2.33 \mathrm{a}$ & $5,010 \mathrm{a}$ & $1.00 \mathrm{a}$ & 113.0 & Early \\
\hline 2011L016 & $"$ & $2.33 \mathrm{a}$ & $4,463 \mathrm{~b}$ & $2.00 \mathrm{~b}$ & 111.0 & Early \\
\hline 2011L029 & $"$ & $2.33 \mathrm{a}$ & $4,637 \mathrm{~b}$ & $1.33 \mathrm{a}$ & 113.0 & Early \\
\hline 2011L129 & $"$ & $2.33 \mathrm{a}$ & $5,124 \mathrm{a}$ & $3.33 \mathrm{c}$ & 125.0 & Average \\
\hline 2011L069 & $"$ & $2.67 \mathrm{a}$ & $3,714 \mathrm{~b}$ & $2.00 \mathrm{~b}$ & 122.3 & Average \\
\hline 2011L155 & $"$ & $2.67 \mathrm{a}$ & $5,401 \mathrm{a}$ & $3.33 \mathrm{c}$ & 132.0 & Late \\
\hline 2011L035 & $"$ & $3.00 \mathrm{a}$ & $4,400 \mathrm{~b}$ & $2.67 \mathrm{c}$ & 114.3 & Early \\
\hline 2011L066 & $"$ & $3.33 \mathrm{a}$ & $3,999 \mathrm{~b}$ & $5.00 \mathrm{~d}$ & 112.0 & Early \\
\hline 2011L071 & $"$ & $3.33 \mathrm{a}$ & $5,246 \mathrm{a}$ & $6.00 \mathrm{e}$ & 118.0 & Early \\
\hline 2011L075 & $"$ & $3.33 \mathrm{a}$ & $4,533 \mathrm{~b}$ & $6.00 \mathrm{e}$ & 116.0 & Early \\
\hline 2011L083 & $"$ & $3.33 \mathrm{a}$ & $4,430 \mathrm{~b}$ & $4.33 \mathrm{~d}$ & 118.0 & Early \\
\hline 2011L114 & $"$ & $3.33 \mathrm{a}$ & $4,262 \mathrm{~b}$ & $2.00 \mathrm{~b}$ & 120.0 & Average \\
\hline 2011L126 & $"$ & $3.33 \mathrm{a}$ & $4,656 \mathrm{~b}$ & $1.33 \mathrm{a}$ & 122.0 & Average \\
\hline 2011L022 & $"$ & $3.67 \mathrm{~b}$ & $4,662 \mathrm{~b}$ & $2.67 \mathrm{c}$ & 111.0 & Early \\
\hline 2011L036 & $"$ & $3.67 \mathrm{~b}$ & $4,048 \mathrm{~b}$ & $1.67 \mathrm{a}$ & 112.7 & Early \\
\hline 2011L045 & $"$ & $3.67 \mathrm{~b}$ & $5,570 \mathrm{a}$ & $2.00 \mathrm{~b}$ & 126.0 & Average \\
\hline 2011L131 & " & $3.67 \mathrm{~b}$ & $4,884 \mathrm{a}$ & $2.67 \mathrm{c}$ & 124.3 & Average \\
\hline P98Y51 & Cultivar & $3.70 \mathrm{~b}$ & $5,487 \mathrm{a}$ & $1.00 \mathrm{a}$ & 130.0 & Late \\
\hline NA7255RR & " & $4.00 \mathrm{~b}$ & $4,113 \mathrm{~b}$ & $1.00 \mathrm{a}$ & 109.3 & Super-early \\
\hline P99R03 & $"$ & $4.00 \mathrm{~b}$ & $4,766 \mathrm{a}$ & $2.33 \mathrm{~b}$ & 140.3 & Late \\
\hline TMG132RR & " & $4.00 \mathrm{~b}$ & $5,002 \mathrm{a}$ & $1.73 \mathrm{a}$ & 130.0 & Late \\
\hline 2011L044 & Lineage & $4.00 \mathrm{~b}$ & $4,253 \mathrm{~b}$ & $6.00 \mathrm{e}$ & 126.0 & Average \\
\hline 2011L048 & " & $4.00 \mathrm{~b}$ & $4,238 \mathrm{~b}$ & $1.33 \mathrm{a}$ & 117.0 & Early \\
\hline 2011L112 & $"$ & $4.00 \mathrm{~b}$ & $4,809 \mathrm{a}$ & $1.00 \mathrm{a}$ & 134.0 & Late \\
\hline 2011L007 & $"$ & $4.33 \mathrm{~b}$ & $4,267 \mathrm{~b}$ & $2.00 \mathrm{~b}$ & 114.0 & Early \\
\hline 2011L061 & $"$ & $4.33 \mathrm{~b}$ & $4,486 \mathrm{~b}$ & $1.00 \mathrm{a}$ & 119.7 & Early \\
\hline 2011L097 & $"$ & $4.33 \mathrm{~b}$ & $4,124 \mathrm{~b}$ & $6.00 \mathrm{e}$ & 122.3 & Average \\
\hline 2011L128 & $"$ & $4.33 \mathrm{~b}$ & $3,985 \mathrm{~b}$ & $6.00 \mathrm{e}$ & 119.0 & Early \\
\hline 2011L133 & $"$ & $4.33 \mathrm{~b}$ & $5,019 \mathrm{a}$ & $6.33 \mathrm{e}$ & 125.0 & Average \\
\hline 2011L137 & $"$ & $4.33 \mathrm{~b}$ & $4,874 \mathrm{a}$ & $1.33 \mathrm{a}$ & 125.0 & Average \\
\hline 2011L148 & $"$ & $4.33 \mathrm{~b}$ & $4,852 \mathrm{a}$ & $3.67 \mathrm{~d}$ & 119.7 & Early \\
\hline 2011L162 & $"$ & $4.50 \mathrm{~b}$ & $4,913 \mathrm{a}$ & $2.33 \mathrm{~b}$ & 131.0 & Late \\
\hline 2011L014 & $"$ & $4.67 \mathrm{~b}$ & $5,027 \mathrm{a}$ & $4.00 \mathrm{~d}$ & 114.0 & Early \\
\hline 2011L023 & $"$ & $4.67 \mathrm{~b}$ & $4,205 \mathrm{~b}$ & $1.33 \mathrm{a}$ & 117.0 & Early \\
\hline 2011L055 & $"$ & $4.67 \mathrm{~b}$ & $5,152 \mathrm{a}$ & $1.00 \mathrm{a}$ & 130.0 & Late \\
\hline 2011L063 & $"$ & $4.67 \mathrm{~b}$ & $3,785 \mathrm{~b}$ & $7.00 \mathrm{e}$ & 120.3 & Average \\
\hline 2011L094 & $"$ & $4.67 \mathrm{~b}$ & $5,723 \mathrm{a}$ & $2.67 \mathrm{c}$ & 118.0 & Early \\
\hline 2011L101 & $"$ & $4.67 \mathrm{~b}$ & $6,147 \mathrm{a}$ & $1.00 \mathrm{a}$ & 127.0 & Average \\
\hline 2011L102 & $"$ & $4.67 \mathrm{~b}$ & $5,898 \mathrm{a}$ & $3.50 \mathrm{c}$ & 126.0 & Average \\
\hline 2011L111 & $"$ & $4.67 \mathrm{~b}$ & $5,614 \mathrm{a}$ & $1.33 \mathrm{a}$ & 133.0 & Late \\
\hline 2011L124 & $"$ & $4.67 \mathrm{~b}$ & $4,599 \mathrm{~b}$ & $2.00 \mathrm{~b}$ & 139.0 & Late \\
\hline 2011L141 & " & $4.67 \mathrm{~b}$ & $4,433 \mathrm{~b}$ & $3.33 \mathrm{c}$ & 125.3 & Average \\
\hline TMG1176RR & Cultivar & $4.83 \mathrm{~b}$ & $4,202 \mathrm{~b}$ & $3.67 \mathrm{~d}$ & 111.0 & Early \\
\hline P98Y11 & " & $5.00 \mathrm{~b}$ & $4,049 \mathrm{~b}$ & $1.50 \mathrm{a}$ & 110.0 & Early \\
\hline 2011L008 & Lineage & $5.00 \mathrm{~b}$ & $3,839 \mathrm{~b}$ & $2.00 \mathrm{~b}$ & 119.7 & Early \\
\hline 2011L013 & " & $5.00 \mathrm{~b}$ & $5,116 \mathrm{a}$ & $3.33 \mathrm{c}$ & 113.7 & Early \\
\hline 2011L046 & $"$ & $5.00 \mathrm{~b}$ & $4,665 \mathrm{~b}$ & $3.67 \mathrm{~d}$ & 120.0 & Average \\
\hline 2011L100 & $"$ & $5.00 \mathrm{~b}$ & $5113.68 \mathrm{a}$ & $2.00 \mathrm{~b}$ & 126.0 & Average \\
\hline 2011L106 & $"$ & $5.00 \mathrm{~b}$ & $4,837 \mathrm{a}$ & $1.00 \mathrm{a}$ & 131.2 & Late \\
\hline 2011L113 & $"$ & $5.00 \mathrm{~b}$ & $4,679 \mathrm{~b}$ & $2.00 \mathrm{~b}$ & 125.0 & Average \\
\hline 2011L127 & $"$ & $5.00 \mathrm{~b}$ & $5,111 \mathrm{a}$ & $1.00 \mathrm{a}$ & 125.0 & Average \\
\hline
\end{tabular}


2011L135

2011L139

2011L147

2011L154

2011L107

2011L024

2011L059

2011L076

2011L117

2011L146

P98Y70

M8230RR

M7908RR

2010L019

2011L092

2011L108

2011L142

2011L145

2011L153

M8527RR

2011L021

2011L042

2011L077

2011L082

2011L086

2011L095

2011L115

2011L134

2011L140

2011L156

2011L161

2011L149

2011L009

2011L011

2011L019

2011L051

2011L065

2011L078

2011L079

AS8380RR

2010L022

2011L104

2011L136

2011L010

2011L017

2011L027

2011L050

2011L056

2011L057

2011L060

2011L070

2011L081

2011L109

2011L110

2011L125

2011L144

2011L160

2011L105

2011L034

2011L049

2011L052

2011L053

2011L062

2011L099
$2,247 \mathrm{~b}$

$4,251 \mathrm{~b}$

5,162 a

4,772 a

$4,527 \mathrm{~b}$

$4,607 \mathrm{~b}$

4,746 a

$4,613 \mathrm{~b}$

$3,535 \mathrm{~b}$

5,133 a

5,340 a

4,299 b

4,267 b

$4,701 \mathrm{~b}$

4,812 a

5,998 a

4,939 a

5,298 a

5,192 a

4,772 a

4,445 b

$4,603 \mathrm{~b}$

$4,256 \mathrm{~b}$

$4,078 \mathrm{~b}$

5,204 a

5,174 a

5,322 a

4,232 b

5,263 a

5,108 a

4,481 b

5,721 a

4,142 b

5,244 a

4,224 b

5,592 a

5,075 a

4,805 a

5,617 a

$3,761 \mathrm{~b}$

5,632 a

$4,431 \mathrm{~b}$

$4,534 \mathrm{~b}$

$4,637 \mathrm{~b}$

$4,148 \mathrm{~b}$

4,638 b

4,991 a

5,087 a

4,316 b

5,067 a

$4,505 \mathrm{~b}$

$4,392 \mathrm{~b}$

$4,461 \mathrm{~b}$

4,768 a

$3,810 \mathrm{~b}$

$4,675 \mathrm{~b}$

5,058 a

4,858 a

$3,532 \mathrm{~b}$

$4,643 \mathrm{~b}$

$4,580 \mathrm{~b}$

4,698 b

$4,165 \mathrm{~b}$

$4,082 \mathrm{~b}$
$1.33 \mathrm{a}$

$2.00 \mathrm{~b}$

$4.00 \mathrm{~d}$

$6.33 \mathrm{e}$

$5.00 \mathrm{~d}$

$4.00 \mathrm{~d}$

$2.00 \mathrm{~b}$

$4.00 \mathrm{~d}$

$1.00 \mathrm{a}$

$1.00 \mathrm{a}$

$1.80 \mathrm{a}$

$4.33 \mathrm{~d}$

$3.33 \mathrm{c}$

$1.08 \mathrm{a}$

$2.67 \mathrm{c}$

$6.33 \mathrm{e}$

$2.00 \mathrm{~b}$

$2.00 \mathrm{~b}$

$1.00 \mathrm{a}$

$2.00 \mathrm{~b}$

$4.00 \mathrm{~d}$

$4.67 \mathrm{~d}$

$2.00 \mathrm{~b}$

$2.00 \mathrm{~b}$

$5.00 \mathrm{~d}$

$2.33 \mathrm{~b}$

$1.33 \mathrm{a}$

$3.33 \mathrm{c}$

$1.00 \mathrm{a}$

$1.00 \mathrm{a}$

$6.00 \mathrm{e}$

$1.33 \mathrm{a}$

$3.67 \mathrm{~d}$

$6.00 \mathrm{e}$

$1.00 \mathrm{a}$

$1.00 \mathrm{a}$

$1.33 \mathrm{a}$

$4.33 \mathrm{~d}$

$7.00 \mathrm{e}$

$3.20 \mathrm{c}$

$2.00 \mathrm{~b}$

$3.67 \mathrm{c}$

$7.00 \mathrm{e}$

$1.00 \mathrm{a}$

$4.00 \mathrm{~d}$

$1.00 \mathrm{a}$

$3.33 \mathrm{c}$

$2.33 \mathrm{~b}$

$2.67 \mathrm{c}$

$4.00 \mathrm{~d}$

$3.33 \mathrm{c}$

$1.00 \mathrm{a}$

$5.00 \mathrm{~d}$

$1.00 \mathrm{a}$

$4.33 \mathrm{~d}$

$7.33 \mathrm{e}$

$1.00 \mathrm{a}$

$4.20 \mathrm{~d}$

$1.00 \mathrm{a}$

$2.33 \mathrm{~b}$

$1.00 \mathrm{a}$

$2.33 \mathrm{~b}$

$2.00 \mathrm{~b}$

$1.33 \mathrm{a}$
135.0

120.3

127.0

124.0

133.6

111.0

127.0

123.0

130.0

125.3

133.0

127.0

119.0

125.0

125.0

122.7

123.3

124.0

127.0

131.0

110.7

122.7

117.0

118.7

128.0

124.0

130.0

122.0

124.3

124.7

134.0

118.7

113.0

114.3

116.3

129.0

124.0

119.3

122.0

127.4

139.0

129.3

129.0

114.0

119.7

112.0

126.0

140.0

140.0

135.0

117.0

121.3

131.0

133.0

140.3

118.7

122.0

129.6

118.0

115.0

130.3

123.0

115.0

124.0

Late

Average

Average

Average

Late

Early

Average

Average

Late

Average

Late

Average

Early

Average

Average

Average

Average

Average

Average

Late

Early

Average

Early

Early

Average

Average

Late

Average

Average

Average

Late 


\begin{tabular}{|c|c|c|c|c|c|c|}
\hline 2011L119 & $"$ & $7.00 \mathrm{c}$ & $5,302 \mathrm{a}$ & $3.33 \mathrm{c}$ & 126.0 & Average \\
\hline 2011L138 & $"$ & $7.00 \mathrm{c}$ & $5,113 \mathrm{a}$ & $1.33 \mathrm{a}$ & 122.0 & Average \\
\hline 2011L018 & $"$ & $7.33 \mathrm{c}$ & $4,050 \mathrm{~b}$ & $2.33 \mathrm{~b}$ & 115.0 & Early \\
\hline 2011L072 & $"$ & $7.33 \mathrm{c}$ & $3,830 \mathrm{~b}$ & $1.00 \mathrm{a}$ & 112.0 & Early \\
\hline 2011L074 & $"$ & $7.33 \mathrm{c}$ & $4,401 \mathrm{~b}$ & $2.33 \mathrm{~b}$ & 123.3 & Average \\
\hline 2011L080 & $"$ & $7.33 \mathrm{c}$ & $4,577 \mathrm{~b}$ & $4.00 \mathrm{~d}$ & 130.0 & Late \\
\hline 2011L085 & $"$ & $7.33 \mathrm{c}$ & $5,067 \mathrm{a}$ & $2.00 \mathrm{~b}$ & 131.0 & Late \\
\hline 2011L089 & $"$ & $7.33 \mathrm{c}$ & $4,673 \mathrm{~b}$ & $1.33 \mathrm{a}$ & 128.7 & Average \\
\hline 2011L151 & $"$ & $7.33 \mathrm{c}$ & $5,885 \mathrm{a}$ & $2.33 \mathrm{~b}$ & 123.0 & Average \\
\hline 2011L158 & $"$ & $7.33 \mathrm{c}$ & $5,064 \mathrm{a}$ & $2.33 \mathrm{~b}$ & 131.3 & Late \\
\hline 2011L118 & $"$ & $7.45 \mathrm{c}$ & $4,293 b$ & $5.33 \mathrm{e}$ & 134.5 & Late \\
\hline 2010L021 & $"$ & $7.67 \mathrm{c}$ & $4,837 \mathrm{a}$ & $4.00 \mathrm{~d}$ & 124.0 & Average \\
\hline 2011L030 & $"$ & $7.67 \mathrm{c}$ & $3,838 \mathrm{~b}$ & $2.00 \mathrm{~b}$ & 114.3 & Early \\
\hline 2011L031 & $"$ & $7.67 \mathrm{c}$ & $4,276 \mathrm{~b}$ & $2.00 \mathrm{~b}$ & 115.7 & Early \\
\hline 2011L043 & $"$ & $7.67 \mathrm{c}$ & $4,545 \mathrm{~b}$ & $2.00 \mathrm{~b}$ & 121.0 & Average \\
\hline 2011L054 & $"$ & $7.67 \mathrm{c}$ & $5,270 \mathrm{a}$ & $1.33 \mathrm{a}$ & 121.0 & Average \\
\hline 2011L064 & $"$ & $7.67 \mathrm{c}$ & $3,620 \mathrm{~b}$ & $1.33 \mathrm{a}$ & 119.0 & Early \\
\hline 2011L088 & $"$ & $7.67 \mathrm{c}$ & $4,048 \mathrm{~b}$ & $1.00 \mathrm{a}$ & 122.0 & Average \\
\hline 2011L093 & $"$ & $7.67 \mathrm{c}$ & $4,421 \mathrm{~b}$ & $4.00 \mathrm{~d}$ & 116.3 & Early \\
\hline 2011L121 & $"$ & $7.67 \mathrm{c}$ & $5,268 \mathrm{a}$ & $4.00 \mathrm{~d}$ & 136.7 & Late \\
\hline 2011L132 & $"$ & $7.67 \mathrm{c}$ & $5,467 \mathrm{a}$ & $1.00 \mathrm{a}$ & 126.7 & Average \\
\hline 2011L143 & $"$ & $7.67 \mathrm{c}$ & $4,292 \mathrm{~b}$ & $3.33 \mathrm{c}$ & 120.3 & Average \\
\hline 2011L157 & $"$ & $7.67 \mathrm{c}$ & $3,867 \mathrm{~b}$ & $2.67 \mathrm{c}$ & 131.0 & Late \\
\hline M9144RR & Cultivar & $7.80 \mathrm{c}$ & $4,743 \mathrm{a}$ & $1.20 \mathrm{a}$ & 140.0 & Late \\
\hline 2010L016 & Lineage & $7.83 \mathrm{c}$ & $5,078 \mathrm{a}$ & $7.62 \mathrm{e}$ & 131.0 & Late \\
\hline 2010L017 & " & $8.00 \mathrm{c}$ & $4,578 \mathrm{~b}$ & $2.00 \mathrm{~b}$ & 115.0 & Early \\
\hline 2011L020 & $"$ & $8.00 \mathrm{c}$ & $3,810 \mathrm{~b}$ & $2.67 \mathrm{c}$ & 115.0 & Early \\
\hline 2011L025 & $"$ & $8.00 \mathrm{c}$ & $4,736 \mathrm{a}$ & $2.33 \mathrm{~b}$ & 113.0 & Early \\
\hline 2011L087 & $"$ & $8.00 \mathrm{c}$ & $4,221 b$ & $1.00 \mathrm{a}$ & 125.3 & Average \\
\hline 2011L096 & $"$ & $8.00 \mathrm{c}$ & $4,552 \mathrm{~b}$ & $1.33 \mathrm{a}$ & 132.0 & Late \\
\hline 2011L098 & $"$ & $8.00 \mathrm{c}$ & $4,230 \mathrm{~b}$ & $1.00 \mathrm{a}$ & 127.0 & Average \\
\hline 2011L103 & $"$ & $8.00 \mathrm{c}$ & $4,779 \mathrm{a}$ & $1.00 \mathrm{a}$ & 123.3 & Average \\
\hline 2011L116 & $"$ & $8.00 \mathrm{c}$ & $5,363 \mathrm{a}$ & $3.66 \mathrm{c}$ & 127.7 & Average \\
\hline 2011L120 & $"$ & $8.00 \mathrm{c}$ & $4,241 \mathrm{~b}$ & $2.67 \mathrm{c}$ & 134.0 & Late \\
\hline 2011L122 & $"$ & $8.00 \mathrm{c}$ & $4,034 \mathrm{~b}$ & $6.00 \mathrm{e}$ & 133.0 & Late \\
\hline 2011L123 & $"$ & $8.00 \mathrm{c}$ & $3,928 \mathrm{~b}$ & $1.00 \mathrm{a}$ & 141.0 & Late \\
\hline 2011L130 & $"$ & $8.00 \mathrm{c}$ & $4,822 \mathrm{a}$ & $3.33 \mathrm{c}$ & 122.0 & Average \\
\hline 2011L028 & $"$ & $8.33 \mathrm{c}$ & $5,173 \mathrm{a}$ & $1.33 \mathrm{a}$ & 116.0 & Early \\
\hline 2011L012 & $"$ & $8.67 \mathrm{c}$ & $4,328 \mathrm{~b}$ & $5.33 \mathrm{e}$ & 122.7 & Average \\
\hline 2011L026 & $"$ & $8.67 \mathrm{c}$ & $5,559 \mathrm{a}$ & $1.33 \mathrm{a}$ & 110.0 & Early \\
\hline 2011L091 & $"$ & $8.67 \mathrm{c}$ & $4,684 \mathrm{~b}$ & $2.67 \mathrm{c}$ & 122.0 & Average \\
\hline 2011L015 & $"$ & $9.00 \mathrm{c}$ & $3,527 \mathrm{~b}$ & $7.00 \mathrm{e}$ & 119.7 & Early \\
\hline CV (\%) & & 33.31 & 25.15 & 15.33 & & \\
\hline
\end{tabular}

Measures followed by the same letter in the column do not differ according to the Scott-Knott test $(\mathrm{P}<0.05)$.

Three groups were formed in Jataí, based on the lodging index, the first with the lowest index, ranging from 1 to 2.33 , and composed of 44 genotypes (Table 2 ). Lodging in the second group, with ten genotypes, varied between 2.67 and 3.67 and the third group had nine genotypes with lodging between 4.00 and 5.50.

Strains 2010L013, 2010L011 and 2011L003 showed a low incidence rate and low lodging index. By contrast, cultivars NA7255RR, AS7307RR and P98Y11 displayed a low lodging index but were more susceptible to the disease. These results indicate that, although the lodging index is a variable that modifies the physiological behavior of the crop, which may lead to a reduction in disease intensity, other factors linked to the genetic base of the plant may be associated with the consistent expression of resistance reactions (YANG et al., 1999; JULIATTI et al., 2013a; ZHAO et al., 2015). Another possible explanation for this phenotypic instability may be the influence of gene expression with quantitative trait effects in the host plant, responsible for modulating the complex resistance of soybean to white mold (VUONG et al., 2008; ZHAO et al., 2015).

It is known that environmental conditions interfere in host physiology. Genotypes that act in different maturity groups, as a function of different agroecosystems, may express different degrees of susceptibility, especially when climatic conditions favor the development of white mold (VIDIC et al., 2013). 
Table 2. Incidence rate of Sclerotinia sclerotiorum, grain yield, lodging index and the cycle of 63 soybean genotypes assessed in Jataí, GO, Brazil in the 2011/2012 growing season.

\begin{tabular}{|c|c|c|c|c|c|c|}
\hline Genotypes & Denomination & Incidence rate & $\begin{array}{l}\text { Yield } \\
\text { kg ha }^{-1}\end{array}$ & Lodging index & Cycle (days) & Classification \\
\hline 2010L013 & Lineage & $1.00 \mathrm{a}$ & $4,971 \mathrm{~b}$ & $1.00 \mathrm{a}$ & 107.3 & Early \\
\hline 2011L003 & " & $1.00 \mathrm{a}$ & $5,150 \mathrm{~b}$ & $1.00 \mathrm{a}$ & 121.0 & Late \\
\hline 2010L011 & " & $1.33 \mathrm{a}$ & $7,484 \mathrm{a}$ & $1.00 \mathrm{a}$ & 108.7 & Early \\
\hline ANTA82 & Cultivar & $1.67 \mathrm{a}$ & $5,498 \mathrm{a}$ & $1.22 \mathrm{a}$ & 117.0 & Average \\
\hline NA7337RR & " & $1.67 \mathrm{a}$ & $4,500 \mathrm{~b}$ & $1.33 \mathrm{a}$ & 123.3 & Late \\
\hline 2011L040 & Lineage & $1.67 \mathrm{a}$ & $5,134 \mathrm{~b}$ & $1.33 \mathrm{a}$ & 112.3 & Average \\
\hline 2010L012 & " & $2.00 \mathrm{a}$ & $5,974 \mathrm{a}$ & $1.00 \mathrm{a}$ & 107.0 & Early \\
\hline 2010L010 & $"$ & $2.33 \mathrm{a}$ & $6,302 \mathrm{a}$ & $1.00 \mathrm{a}$ & 106.7 & Early \\
\hline 2011L005 & $"$ & $2.33 \mathrm{a}$ & $5,701 \mathrm{a}$ & $1.00 \mathrm{a}$ & 126.7 & Late \\
\hline 2011L019 & $"$ & $2.33 \mathrm{a}$ & $4,833 \mathrm{~b}$ & $1.33 \mathrm{a}$ & 116.7 & Average \\
\hline 2011L041 & $"$ & $2.33 \mathrm{a}$ & $6,123 \mathrm{a}$ & $4.33 \mathrm{c}$ & 117.7 & Average \\
\hline 2011L058 & $"$ & $2.33 \mathrm{a}$ & $5,504 \mathrm{a}$ & $4.33 \mathrm{c}$ & 113.7 & Average \\
\hline 2011L006 & " & $3.00 \mathrm{~b}$ & $4,778 \mathrm{~b}$ & $1.33 \mathrm{a}$ & 123.0 & Late \\
\hline POTENCIARR & Cultivar & $3.33 \mathrm{~b}$ & $6,402 \mathrm{a}$ & $2.67 \mathrm{~b}$ & 115.0 & Average \\
\hline 2011L014 & Lineage & $3.33 \mathrm{~b}$ & $4,618 b$ & $1.33 \mathrm{a}$ & 121.3 & Late \\
\hline V-MAXRR & Cultivar & $3.67 \mathrm{~b}$ & $6,549 a$ & $1.33 \mathrm{a}$ & 113.7 & Average \\
\hline 2010L015 & Lineage & $3.67 \mathrm{~b}$ & $6,321 \mathrm{a}$ & $2.00 \mathrm{a}$ & 113.7 & Average \\
\hline 2011L038 & " & $3.67 \mathrm{~b}$ & $5,893 \mathrm{a}$ & $1.00 \mathrm{a}$ & 109.7 & Early \\
\hline 2011L073 & $"$ & $3.67 \mathrm{~b}$ & $3,438 \mathrm{~b}$ & $2.00 \mathrm{a}$ & 136.3 & Late \\
\hline 2011L022 & $"$ & $4.00 \mathrm{~b}$ & $5,184 \mathrm{~b}$ & $1.33 \mathrm{a}$ & 120.7 & Late \\
\hline 2011L033 & $"$ & $4.00 \mathrm{~b}$ & $6,970 \mathrm{a}$ & $2.00 \mathrm{a}$ & 110.3 & Average \\
\hline 2011L004 & $"$ & $4.33 \mathrm{~b}$ & $5,464 \mathrm{a}$ & $1.00 \mathrm{a}$ & 123.7 & Late \\
\hline 2011L021 & $"$ & $4.33 \mathrm{~b}$ & $4,756 \mathrm{~b}$ & $2.00 \mathrm{a}$ & 120.3 & Late \\
\hline 2011L039 & $"$ & $4.33 \mathrm{~b}$ & $4,376 \mathrm{~b}$ & $1.33 \mathrm{a}$ & 123.7 & Late \\
\hline 2011L068 & $"$ & $4.33 \mathrm{~b}$ & $5,814 \mathrm{a}$ & $4.00 \mathrm{c}$ & 116.3 & Average \\
\hline 2011L157 & $"$ & $4.33 \mathrm{~b}$ & $5,029 \mathrm{~b}$ & $2.67 \mathrm{~b}$ & 113.7 & Average \\
\hline 2011L024 & $"$ & $4.67 \mathrm{c}$ & $4,241 \mathrm{~b}$ & $1.33 \mathrm{a}$ & 118.3 & Average \\
\hline 2011L037 & " & $4.67 \mathrm{c}$ & $6,297 \mathrm{a}$ & $2.33 \mathrm{a}$ & 117.0 & Average \\
\hline M7211RR & Cultivar & $5.00 \mathrm{c}$ & $5,132 \mathrm{~b}$ & $1.33 \mathrm{a}$ & 113.7 & Average \\
\hline 2011L013 & Lineage & $5.00 \mathrm{c}$ & $5,480 \mathrm{a}$ & $1.33 \mathrm{a}$ & 118.7 & Average \\
\hline 2011L090 & " & $5.00 \mathrm{c}$ & $5,819 \mathrm{a}$ & $4.33 \mathrm{c}$ & 117.3 & Average \\
\hline NA7255RR & Cultivar & $5.33 \mathrm{c}$ & $4,458 \mathrm{~b}$ & $1.00 \mathrm{a}$ & 116.7 & Average \\
\hline 2011L008 & Lineage & $5.33 \mathrm{c}$ & $4,187 \mathrm{~b}$ & $2.33 \mathrm{a}$ & 128.3 & Late \\
\hline 2011L020 & " & $5.33 \mathrm{c}$ & $4,667 \mathrm{~b}$ & $1.00 \mathrm{a}$ & 119.0 & Average \\
\hline 2011L023 & $"$ & $5.33 \mathrm{c}$ & $4,330 \mathrm{~b}$ & $1.67 \mathrm{a}$ & 125.3 & Late \\
\hline 2011L028 & " & $5.33 \mathrm{c}$ & $4,197 \mathrm{~b}$ & $3.67 \mathrm{~b}$ & 127.3 & Late \\
\hline M7639RR & Cultivar & $5.67 \mathrm{c}$ & $4,226 \mathrm{~b}$ & $3.33 \mathrm{~b}$ & 118.7 & Average \\
\hline 2011L015 & Lineage & $5.67 \mathrm{c}$ & $4,794 \mathrm{~b}$ & $3.33 \mathrm{~b}$ & 128.0 & Late \\
\hline 2011L016 & " & $5.67 \mathrm{c}$ & $4,952 \mathrm{~b}$ & $1.33 \mathrm{a}$ & 118.7 & Average \\
\hline 2011L047 & " & $5.67 \mathrm{c}$ & $4,829 \mathrm{~b}$ & $3.33 \mathrm{~b}$ & 118.7 & Average \\
\hline TMG1176RR & Cultivar & $6.00 \mathrm{c}$ & $4,443 \mathrm{~b}$ & $4.50 \mathrm{c}$ & 121.5 & Late \\
\hline TMG-123RR & " & $6.00 \mathrm{c}$ & $5,672 \mathrm{a}$ & $5.50 \mathrm{c}$ & 113.5 & Average \\
\hline 2011L011 & Lineage & $6.00 \mathrm{c}$ & $4,385 \mathrm{~b}$ & $2.67 \mathrm{~b}$ & 118.7 & Average \\
\hline M8230 RR & Cultivar & $6.17 \mathrm{c}$ & $2,839 \mathrm{~b}$ & $2.83 \mathrm{~b}$ & 138.0 & Late \\
\hline P98Y11 & " & $6.33 \mathrm{c}$ & $4,496 \mathrm{~b}$ & $1.17 \mathrm{a}$ & 119.7 & Average \\
\hline 2011L012 & Lineage & $6.33 \mathrm{c}$ & $3,979 \mathrm{~b}$ & $3.67 \mathrm{~b}$ & 128.0 & Late \\
\hline 2011L018 & " & $6.33 \mathrm{c}$ & $4,504 \mathrm{~b}$ & $1.67 \mathrm{a}$ & 124.0 & Late \\
\hline 2011L029 & $"$ & $6.33 \mathrm{c}$ & $4,376 \mathrm{~b}$ & $1.67 \mathrm{a}$ & 122.7 & Late \\
\hline 2010L017 & $"$ & $6.50 \mathrm{c}$ & $4,473 \mathrm{~b}$ & $2.17 \mathrm{a}$ & 129.3 & Late \\
\hline 2010L005 & “ & $6.67 \mathrm{c}$ & $5,956 \mathrm{a}$ & $1.00 \mathrm{a}$ & 113.3 & Average \\
\hline 2011L010 & $"$ & $6.67 \mathrm{c}$ & $3,431 \mathrm{~b}$ & $4.00 \mathrm{c}$ & 129.0 & Late \\
\hline 2011L027 & $"$ & $6.67 \mathrm{c}$ & $3,644 \mathrm{~b}$ & $1.67 \mathrm{a}$ & 128.7 & Late \\
\hline 2010L021 & $"$ & $7.00 \mathrm{c}$ & $3,600 \mathrm{~b}$ & $4.33 \mathrm{c}$ & 135.0 & Late \\
\hline 2011L007 & $"$ & $7.00 \mathrm{c}$ & $4,461 \mathrm{~b}$ & $2.00 \mathrm{a}$ & 125.3 & Late \\
\hline 2011L017 & $"$ & $7.00 \mathrm{c}$ & $4,968 \mathrm{~b}$ & $1.33 \mathrm{a}$ & 127.3 & Late \\
\hline 2011L025 & $"$ & $7.00 \mathrm{c}$ & $3,710 \mathrm{~b}$ & $3.00 \mathrm{~b}$ & 127.3 & Late \\
\hline 2011L026 & $"$ & $7.00 \mathrm{c}$ & $4,262 \mathrm{~b}$ & $4.67 \mathrm{c}$ & 127.0 & Late \\
\hline 2011L031 & " & $7.00 \mathrm{c}$ & $3,966 \mathrm{~b}$ & $1.67 \mathrm{a}$ & 127.0 & Late \\
\hline AS7307RR & Cultivar & $7.33 \mathrm{c}$ & $6,635 a$ & $1.00 \mathrm{a}$ & 113.7 & Average \\
\hline 2011L009 & Lineage & $7.33 \mathrm{c}$ & $4,857 \mathrm{~b}$ & $1.00 \mathrm{a}$ & 120.3 & Late \\
\hline 2011L067 & " & $7.33 \mathrm{c}$ & $4,995 \mathrm{~b}$ & $1.00 \mathrm{a}$ & 120.7 & Late \\
\hline M7908RR & Cultivar & $7.50 \mathrm{c}$ & $3,805 \mathrm{~b}$ & $2.00 \mathrm{a}$ & 125.8 & Late \\
\hline 2011L030 & Lineage & $8.00 \mathrm{c}$ & $3,198 \mathrm{~b}$ & $2.00 \mathrm{a}$ & 127.0 & Late \\
\hline $\mathrm{CV}(\%)$ & & 50.9 & 34.54 & 21.04 & & \\
\hline
\end{tabular}

Measures followed by the same letter in the column do not differ according to the Scott-Knott test $(\mathrm{P}<0.05)$. 
Assessment of yield in Jataí separated genotypes into two groups. Yield ranged from $5,764 \mathrm{~kg} \mathrm{ha}^{-1}$ to $7,484 \mathrm{~kg} \mathrm{ha}^{-1}$ in the first group and $2,839 \mathrm{~kg} \mathrm{ha}^{-1}$ to $5,184 \mathrm{~kg} \mathrm{ha}^{-1}$ in the second. Strain 2010L011, which was early, showed low lodging and incidence rates, but high yield, representing promising material for the region. The cultivar M8230RR produced the lowest yield $\left(2,839 \mathrm{~kg} \mathrm{ha}^{-1}\right)$, likely due to the high incidence (6.17) of the disease.

The commercial cultivars with the highest yields were AS7307RR, V-MAX-RR, and POTENCIA-RR, with average yields of $6,635,6,549$ and $6,402 \mathrm{~kg} \mathrm{ha}^{-1}$, respectively, not statistically different from TMG123RR and ANTA82.

Analysis of 37 genotypes planted in both areas (Barreiras/BA, and Jataí/GO) showed no significant interaction between the area and genotype concerning white mold incidence. The most resistant group contained five genotypes, ANTA82, 2011L003, NA7337RR, 2011L005, and 2011L006, since it maintained the degree of resistance when planted in both regions (Table 3 ).

Table 3. Incidence rate of Sclerotinia sclerotiorum, grain yield, lodging index and the cycle of 37 soybean genotypes assessed in Barreiras. BA and Jataí. Go in the 2011/2012 growing season.

\begin{tabular}{|c|c|c|c|c|c|c|c|c|}
\hline \multirow{2}{*}{$\begin{array}{l}\text { Cultivars or } \\
\text { Lineage }\end{array}$} & \multicolumn{2}{|c|}{ Incidence rate } & \multicolumn{2}{|c|}{ Lodging index } & \multicolumn{2}{|c|}{ Cycle } & \multicolumn{2}{|c|}{ Yield $\left(\mathrm{kg} \mathrm{ha}^{-1}\right)$} \\
\hline & Barreiras & Jataí & Barreiras & Jataí & Barreiras & Jataí & Barreiras & Jataí \\
\hline 2011L003 & $1.67 \mathrm{a} \mathrm{A}$ & $1.00 \mathrm{a} \mathrm{A}$ & $1.67 \mathrm{a} \mathrm{A}$ & $1.00 \mathrm{a} \mathrm{A}$ & 110.0 & 121.0 & 4,756 a $\mathrm{A}$ & $5,150 \mathrm{~b} \mathrm{~A}$ \\
\hline ANTA82 & $1.11 \mathrm{a} \mathrm{A}$ & $1.67 \mathrm{a} \mathrm{A}$ & $1.00 \mathrm{a} A$ & $1.22 \mathrm{a} \mathrm{A}$ & 101.7 & 117.0 & $4,522 \mathrm{~b} \mathrm{~A}$ & 5,499 a $\mathrm{A}$ \\
\hline NA7337RR & $1.67 \mathrm{a} \mathrm{A}$ & $1.67 \mathrm{a} A$ & $1.00 \mathrm{a} \mathrm{A}$ & $1.33 \mathrm{a} \mathrm{A}$ & 108.0 & 123.3 & $4,311 \mathrm{~b} \mathrm{~A}$ & $4,500 \mathrm{~b} \mathrm{~A}$ \\
\hline 2011L005 & $2.33 \mathrm{a} \mathrm{A}$ & 2.33 a A & $1.00 \mathrm{a} \mathrm{A}$ & $1.00 \mathrm{a} \mathrm{A}$ & 113.0 & 126.7 & $4,635 \mathrm{~b} \mathrm{~A}$ & 5,701 a B \\
\hline 2011L019 & $6.33 \mathrm{c} \mathrm{A}$ & 2.33 a B & $1.00 \mathrm{a} \mathrm{A}$ & $1.33 \mathrm{a} \mathrm{A}$ & 116.3 & 116.7 & $4,224 \mathrm{~b} \mathrm{~A}$ & $4,833 \mathrm{~b} \mathrm{~A}$ \\
\hline 2011L006 & $2.33 \mathrm{a} \mathrm{A}$ & $3.00 \mathrm{~b} \mathrm{~A}$ & $1.00 \mathrm{a} \mathrm{A}$ & $1.33 \mathrm{a} \mathrm{A}$ & 113.0 & 123.0 & 5,010 a A & $4,778 \mathrm{~b} \mathrm{~A}$ \\
\hline 2011L014 & $4.67 \mathrm{~b} \mathrm{~A}$ & $3.33 \mathrm{~b} \mathrm{~A}$ & $4.00 \mathrm{~d} \mathrm{~A}$ & $1.33 \mathrm{a} \mathrm{A}$ & 114.0 & 121.3 & 5,027 a A & $4,618 \mathrm{~b} \mathrm{~A}$ \\
\hline 2011L022 & $3.67 \mathrm{~b} \mathrm{~A}$ & $4.00 \mathrm{~b} \mathrm{~A}$ & $2.67 \mathrm{c} \mathrm{A}$ & $1.33 \mathrm{a} \mathrm{A}$ & 110.7 & 120.7 & $4,662 \mathrm{~b} \mathrm{~A}$ & 5,184 a A \\
\hline 2011L021 & $6.00 \mathrm{c} \mathrm{A}$ & $4.33 \mathrm{~b} \mathrm{~B}$ & $4.00 \mathrm{~d} \mathrm{~A}$ & $2.00 \mathrm{a} \mathrm{B}$ & 110.7 & 120.3 & $4,445 \mathrm{~b} \mathrm{~A}$ & $4,756 \mathrm{~b} \mathrm{~A}$ \\
\hline 2011L024 & $5.33 \mathrm{~b} \mathrm{~A}$ & $4.67 \mathrm{c} \mathrm{A}$ & $4.00 \mathrm{~d} \mathrm{~A}$ & $1.33 \mathrm{a} \mathrm{A}$ & 111.0 & 118.3 & $4,607 \mathrm{~b} \mathrm{~A}$ & $4,241 \mathrm{~b} \mathrm{~A}$ \\
\hline 2011L013 & $5.00 \mathrm{~b} \mathrm{~A}$ & $5.00 \mathrm{c} \mathrm{A}$ & $3.33 \mathrm{c} \mathrm{A}$ & $1.33 \mathrm{a} \mathrm{A}$ & 113.0 & 118.3 & 5,116 a A & 5,480 a $\mathrm{A}$ \\
\hline 2011L028 & 8.33 c A & $5.33 \mathrm{c} \mathrm{B}$ & $1.33 \mathrm{a} \mathrm{A}$ & $3.67 \mathrm{~b} \mathrm{~B}$ & 116.0 & 127.3 & 5,173 a $\mathrm{A}$ & $4,197 \mathrm{~b}$ A \\
\hline 2011L023 & $4.67 \mathrm{~b} \mathrm{~A}$ & $5.33 \mathrm{c} \mathrm{A}$ & $1.33 \mathrm{a} \mathrm{A}$ & $1.67 \mathrm{a} \mathrm{A}$ & 117.0 & 125.3 & $4,205 \mathrm{~b} \mathrm{~A}$ & $4,330 \mathrm{~b} \mathrm{~A}$ \\
\hline 2011L008 & $5.00 \mathrm{~b} \mathrm{~A}$ & $5.33 \mathrm{c} \mathrm{A}$ & $2.00 \mathrm{~b} \mathrm{~A}$ & $2.33 \mathrm{a} \mathrm{A}$ & 119.7 & 128.3 & $3,839 \mathrm{~b} \mathrm{~A}$ & $4,187 \mathrm{~b} \mathrm{~A}$ \\
\hline NA7255RR & $4.00 \mathrm{~b} \mathrm{~A}$ & $5.33 \mathrm{c} \mathrm{A}$ & $1.00 \mathrm{a} \mathrm{A}$ & $1.00 \mathrm{a} \mathrm{A}$ & 109.3 & 116.7 & $4,113 \mathrm{~b} \mathrm{~A}$ & $4,458 \mathrm{~b} \mathrm{~A}$ \\
\hline 2011L020 & $8.00 \mathrm{c} \mathrm{A}$ & $5.33 \mathrm{c} \mathrm{B}$ & $2.67 \mathrm{c} \mathrm{A}$ & $1.00 \mathrm{a} \mathrm{A}$ & 115.0 & 119.0 & $3,810 \mathrm{~b} \mathrm{~A}$ & $4,667 \mathrm{~b} \mathrm{~A}$ \\
\hline 2011L016 & $2.33 \mathrm{a} \mathrm{A}$ & $5.67 \mathrm{c} \mathrm{B}$ & $2.00 \mathrm{~b} \mathrm{~A}$ & $1.33 \mathrm{a} \mathrm{A}$ & 111.0 & 118.3 & $4,463 \mathrm{~b} \mathrm{~A}$ & $4,951 \mathrm{~b} \mathrm{~A}$ \\
\hline 2011L015 & $9.00 \mathrm{c} \mathrm{A}$ & $5.67 \mathrm{c} \mathrm{B}$ & 7.00 e $\mathrm{A}$ & $3.33 \mathrm{~b} \mathrm{~B}$ & 119.7 & 128.0 & $3,527 \mathrm{~b} \mathrm{~A}$ & 4,794 b B \\
\hline 2011L011 & 6.33 c A & $6.00 \mathrm{c} \mathrm{A}$ & 6.00 e $\mathrm{A}$ & $2.67 \mathrm{~b} \mathrm{~B}$ & 114.3 & 118.3 & 5,244 a A & $4,384 \mathrm{~b} \mathrm{~A}$ \\
\hline TMG1176RR & $4.83 \mathrm{~b} \mathrm{~A}$ & $6.00 \mathrm{c} \mathrm{B}$ & $3.67 \mathrm{~d} \mathrm{~A}$ & $4.50 \mathrm{c} \mathrm{A}$ & 110.7 & 121.5 & $4,202 \mathrm{~b} \mathrm{~A}$ & 4,442 b A \\
\hline P98Y11 & $5.00 \mathrm{~b} \mathrm{~A}$ & $6.33 \mathrm{c} \mathrm{A}$ & $1.50 \mathrm{a} \mathrm{A}$ & 1.17 a A & 110.0 & 119.7 & $4,049 \mathrm{~b} \mathrm{~A}$ & 4,496 b A \\
\hline 2011L018 & 7.33 с A & $6.33 \mathrm{c} \mathrm{A}$ & $2.33 \mathrm{~b} \mathrm{~A}$ & $1.67 \mathrm{a} \mathrm{A}$ & 115.0 & 124.0 & $4,050 \mathrm{~b} \mathrm{~A}$ & $4,504 \mathrm{~b} \mathrm{~A}$ \\
\hline 2011L012 & 8.67 c A & $6.33 \mathrm{c} \mathrm{A}$ & 5.33 e $\mathrm{A}$ & $3.67 \mathrm{~b} \mathrm{~B}$ & 122.7 & 128.0 & $4,328 \mathrm{~b} \mathrm{~A}$ & $3,979 \mathrm{~b} \mathrm{~A}$ \\
\hline 2011L029 & $2.33 \mathrm{a} \mathrm{A}$ & $6.33 \mathrm{c} \mathrm{B}$ & $1.00 \mathrm{a} \mathrm{A}$ & 1.67 a A & 113.0 & 122.7 & $4,637 \mathrm{~b} \mathrm{~A}$ & $4,376 \mathrm{~b} \mathrm{~A}$ \\
\hline 2010L017 & $8.00 \mathrm{c} \mathrm{A}$ & $6.50 \mathrm{c} \mathrm{A}$ & $2.00 \mathrm{~b} \mathrm{~A}$ & 2.17 a A & 115.0 & 129.3 & $4,578 \mathrm{~b} \mathrm{~A}$ & $4,473 \mathrm{~b} \mathrm{~A}$ \\
\hline 2011L027 & $6.67 \mathrm{c} \mathrm{A}$ & $6.67 \mathrm{c} \mathrm{A}$ & $1.33 \mathrm{a} \mathrm{A}$ & $1.67 \mathrm{a} \mathrm{A}$ & 112.0 & 128.7 & $4,638 \mathrm{~b} \mathrm{~A}$ & 3,644 b B \\
\hline 2011L010 & $6.67 \mathrm{c} \mathrm{A}$ & $6.67 \mathrm{c} \mathrm{A}$ & $1.00 \mathrm{a} \mathrm{A}$ & $4.00 \mathrm{c} \mathrm{B}$ & 114.0 & 129.0 & $4,637 \mathrm{~b} \mathrm{~A}$ & $3,431 \mathrm{~b} \mathrm{~A}$ \\
\hline 2011L026 & $8.67 \mathrm{c} \mathrm{A}$ & $7.00 \mathrm{c} \mathrm{A}$ & $2.33 \mathrm{~b} \mathrm{~A}$ & $4.67 \mathrm{c} \mathrm{B}$ & 110.0 & 127.0 & 5,559 a $\mathrm{A}$ & 4,262 b A \\
\hline 2011L031 & $7.67 \mathrm{c} \mathrm{A}$ & $7.00 \mathrm{c} \mathrm{A}$ & $1.33 \mathrm{a} \mathrm{A}$ & $1.67 \mathrm{a} \mathrm{A}$ & 115.7 & 127.0 & $4,276 \mathrm{~b} \mathrm{~A}$ & $3,966 \mathrm{~b} \mathrm{~A}$ \\
\hline 2011L025 & $8.00 \mathrm{c} \mathrm{A}$ & $7.00 \mathrm{c} \mathrm{A}$ & $1.33 \mathrm{a} \mathrm{A}$ & $3.00 \mathrm{~b} \mathrm{~B}$ & 113.0 & 127.3 & 4,736 a $\mathrm{A}$ & $3,710 \mathrm{~b} \mathrm{~A}$ \\
\hline 2011L017 & $6.67 \mathrm{c} \mathrm{A}$ & $7.00 \mathrm{c} \mathrm{A}$ & $4.00 \mathrm{~d} \mathrm{~A}$ & $1.33 \mathrm{a} \mathrm{A}$ & 119.7 & 127.3 & 4,148 b A & $4,968 \mathrm{~b} \mathrm{~A}$ \\
\hline 2010L021 & $7.67 \mathrm{c} \mathrm{A}$ & $7.00 \mathrm{c} \mathrm{A}$ & $4.00 \mathrm{~d} \mathrm{~A}$ & $4.33 \mathrm{c} \mathrm{A}$ & 124.0 & 135.0 & 4,836 a $\mathrm{A}$ & $3,600 \mathrm{~b} \mathrm{~B}$ \\
\hline 2011L007 & $4.33 \mathrm{~b} \mathrm{~A}$ & $7.00 \mathrm{c} \mathrm{B}$ & $2.00 \mathrm{~b} \mathrm{~A}$ & $2.00 \mathrm{a} \mathrm{A}$ & 114.0 & 125.3 & $4,267 \mathrm{~b} \mathrm{~A}$ & $4,461 \mathrm{~b} \mathrm{~A}$ \\
\hline 2011L007 & $4.33 \mathrm{~b} \mathrm{~A}$ & $7.00 \mathrm{c} \mathrm{B}$ & $2.00 \mathrm{~b} \mathrm{~A}$ & $2.00 \mathrm{a} \mathrm{A}$ & 114.0 & 125.3 & $4,267 \mathrm{~b} \mathrm{~A}$ & $4,461 \mathrm{~b} \mathrm{~A}$ \\
\hline 2011L009 & $6.33 \mathrm{c} \mathrm{A}$ & $7.33 \mathrm{c} \mathrm{A}$ & $3.67 \mathrm{~d} \mathrm{~A}$ & $1.00 \mathrm{a} \mathrm{B}$ & 113.0 & 120.3 & $4,142 \mathrm{~b} \mathrm{~A}$ & $4,857 \mathrm{~b} \mathrm{~A}$ \\
\hline M7908RR & $5.67 \mathrm{c} \mathrm{A}$ & $7.50 \mathrm{c} \mathrm{B}$ & $3.33 \mathrm{c} \mathrm{A}$ & $2.00 \mathrm{a} \mathrm{A}$ & 119.0 & 125.3 & $4,267 \mathrm{~b} \mathrm{~A}$ & $3,805 \mathrm{~b} \mathrm{~A}$ \\
\hline 2011L030 & 7.67 c A & $8.00 \mathrm{c} \mathrm{A}$ & $1.33 \mathrm{a} \mathrm{A}$ & $2.00 \mathrm{a} \mathrm{A}$ & 114.3 & 127.0 & $3,838 \mathrm{~b} \mathrm{~A}$ & 3,198 b A \\
\hline CV $(\%)$ & & & & & & & & \\
\hline
\end{tabular}

Measures followed by the same lower-case letter in the column and upper case on the line do not differ according to the Scott-Knott test (P<0.05). Considering each variable analyzed. 
A significant positive correlation was observed between lodging and disease incidence and between genotype cycle and disease incidence, that is, the larger the lodging index and crop cycle, the higher the incidence of the disease. Pearson's coefficient showed a significant negative correlation between soybean yield and disease incidence, that is, the higher the incidence, the lower the yield (Table 4).

For most genotypes, the lodging index was higher for Barreiras, and the cycles of all the strains were higher in Jataí than in Barreiras. About yield, two strains, 2011L021 and 2011L027 increased in Barreiras, with a rise of $27 \%$ and $34 \%$, respectively. Strains 2011L005 and 2011L015 showed a yield increase in
Jataí, with a rise of 23 and $36 \%$, respectively, which demonstrates adaptability and stability in different regions.

As observed in this study, 29 genotypes maintained their incidence levels at the different study sites. Some authors have reported partial resistance expression in field assessments (GRAU; RADKE, 1984; WEGULO et al., 1998; KIM et al., 1999; YANG et al., 1999). Studies conducted by Kim e Diers (2000) show significant genotype-environment interaction in populations of soybean genotypes in four environments in Michigan, USA. These authors found that the reaction of cultivars to $S$. sclerotiorum may be influenced by environmental factors, similar to what was observed here.

Table 4. Pearson's correlation between the variables: local, incidence rate, lodging index, crop cycle (days) and yield (kg ha $\left.{ }^{-1}\right)$

\begin{tabular}{|c|c|c|c|c|c|}
\hline & Local & Incidence & Lodging & Cycle & Yield \\
\hline Local & 1 & & & & \\
\hline Incidence & $0.002^{\mathrm{ns}}$ & 1 & & & \\
\hline Lodging & $-0.131^{\mathrm{ns}}$ & $0.379 * *$ & 1 & & \\
\hline Cycle & $0.716^{* *}$ & $0.346^{* *}$ & $0.225^{\mathrm{ns}}$ & 1 & \\
\hline Yield & $-0.058^{\mathrm{ns}}$ & $-0.409^{* *}$ & $-0.249^{\mathrm{ns}}$ & $-0.376^{\mathrm{ns}}$ & 1 \\
\hline
\end{tabular}

** Significant $(\mathrm{P}<0.05) ;{ }^{\mathrm{ns}}$ Not significant $(\mathrm{P}>0.05)$.

Genetic evidence of physiological resistance and prevention mechanisms against white mold was described by Kim e Diers (2000) in the mapping of three QTL genes. Two loci are related to prevention mechanisms and are primarily associated with flowering time, plant height and lodging index. The third locus likely accounts for the partial physiological resistance of the plant. Most studies show that at least four different genomic regions are involved in the plant resistance response to soybean white mold (ZHAO et al., 2015).

\section{Conclusions}

In different agroecosystems, the environment influenced the cultivar maturation cycle in the two areas, in addition to the significant interaction observed between disease incidence and both the cycle and yield.

In Barreiras, BA, the cultivar M7639RR and strains 2011L003, 2011L006, 2011L129, and 2011L155 showed high resistance, high yield and low lodging index, which are promising signs for the region.

In Jataí, GO the cultivar ANTA82 and strains 2010L011, 2010L010, 2010L012 and 2011L005 were selected for their high yield, low disease incidence, and low lodging index.

Genotypes 2011L005, 2011L003, 2011L006, NA7337RR, and ANTA82, exhibited stability with low disease incidence, low lodging index, and high yield.

\section{References}

BERNARD, R. L.; CHAMBERLAIN, D. W.; LAWRENCE, R. D. Result of the cooperative uniform soybeans tests. Washington: USDA, 1965. $132 \mathrm{p}$.

BOLAND, G. J.; HALL, R. Evaluating soybean cultivars for resistance to Sclerotinia sclerotiorum under field conditions. Plant Disease, Saint Paul, v. 71, n. 10, p. 934-936, 1987.

BOLTON, M. D.; THOMMA, B. P. H. J.; NELSON, B. D. Sclerotinia sclerotiorum (Lib.) de Bary: biology and molecular traits of a cosmopolitan pathogen. Molecular Plant Pathology, Londres, v. 7, n. 1, p. 1-16, 2006.

CONAB. COMPANHIA NACIONAL DE ABASTECIMENTO. Acompanhamento da safra brasileira de grãos: safra 2014/15. Brasília-DF: CONAB/ Ministério do Planejamento, Orçamento e Gestão, 2015. 119 p.

CUNHA, W. G.; TINOCO, M. L. P.; PANCOTI, H. L.; RIBEIRO, R. E.; ARAGÃO, F. J. L. High resistance to Sclerotinia sclerotiorum in transgenic soybean plants transformed to express an oxalate decarboxylase gene. Plant Pathology, Hoboken, v. 59, n. 4, p. 654 660, 2010.

FEHR, W. R.; CAVINESS, C. E.; BURMOOD, D. T.; PENNINGTON, J. S. Stage of development descriptions for soybeans, Glycine max (L.) Merrill. Crop Science, Madison, v. 11, n. 6 , p. $929-931,1971$.

FERREIRA, D. F. Sisvar: a computer statistical analysis system. Ciência e Agrotecnologia, Lavras-MG, v. 35, n. 6, p. 1039-1042, 2011.

GARCiA, R. A.; MEYER, M. C.; ÁVILA, K. A. G. B., CUNHA, M.G. Métodos de inoculação de Sclerotinia sclerotiorum para triagem de cultivares de soja resistentes ao 
mofo-branco. Pesquisa Agropecuária Brasileira, BrasíliaDF, v. 50, n. 8, p. 726-729, 2015.

GRAU, C. R.; RADKE, V. L. Effects of cultivars and cultural practices on sclerotinia stem rot of soybean. Plant Disease, Saint Paul, v. 68, n. 1, p. 56-58, 1984.

HOFFMAN, D. D.; DIERS, B. W.; HARTMAN, G. L.; NICKELL, C. D.; NELSON, R. L.; PEDERSEN, W. L.; COBER, E. R.; GRAEF, G. L.; STEADMAN, J. R.; GRAU, C. R.; NELSON, B. D.; DEL RIO, L. E.; HELMS, T.; ANDERSON, T.; POYSA, V.; RAJCAN, I.; STIENSTRA, W. C. Selected soybean plant introductions with partial resistance to Sclerotinia sclerotiorum. Plant Disease, Saint Paul, v. 86, n. 9, p. 971-980, 2002.

JULIATTI, F. C.; CAIRES, A. M.; JULIATTI, B. C. M.; BORIN, M. R.; SOUZA FILHO, D. J. Reação de genótipos de soja transgênicos e convencionais à podridão branca da haste. Bioscience Journal, Uberlândia-MG, v. 29, n. 4, p. 921-931, 2013a.

JUliatTi, F. C.; SAGATA, E.; JULiATTI, B. C. M. Ranqueamento de genótipos de soja com resistência parcial por diferentes métodos de inoculação de Sclerotinia Sclerotiorum submetidos à análise de correlação. Bioscience Journal, Uberlândia-MG, v. 29, n. 3, p. 681-689, 2013 b.

KIM, H. S.; DIERS, B. W. Inheritance of partial resistance to sclerotinia stem rot in soybean. Crop Science, Madison, v. 40, n. 1, p. 55-61, 2000.

KIM, H. S.; SNELLER, C. H.; DIERS, B. W. Evaluation of soybean cultivars for resistance to sclerotinia stem rot in field environments. Crop Science, Madison, v. 39, n. 1, p. 64-68, 1999.
KURLE, J. E.; GRAU, C. R.; OPLINGER, E. S.; MENGISTU, A. Tillage, crop sequence, and cultivar effect on sclerotinia stem rot incidence and yield in soybean. Agronomy Journal, Madison, v. 93, n. 5, p. 973-982, 2001.

VIDIC, M.; DORDEVIC, V.; PETROVIC, K.; MILADINOVIC, J. Review of soybean resistance to pathogens. Field and Vegetable Crops Research, Sérvia, v. 50, n. 2, p. 52-61, 2013.

VUONG, T. D., DIERS, B. W. AND HARTMAN, G. L. Identification of QTL for resistance to sclerotinia stem rot in soybean plant introduction 194639. Crop Science, Madison, v. 48, n. 6, p. 2209-2214, 2008.

WEGUlO, S. N.; YANG, X. B.; MARTINSON, C. A. Soybean cultivar responses to Sclerotinia sclerotiorum in field and controlled environment studies. Plant Disease, Saint Paul, v. 82 , n. 11 , p. 1264-1270, 1998.

WRATHER, A.; SHANNON, G.; BALARDIN, R.; CARREGAL, L.; ESCOBAR, R.; GUPTA, G. K.; MA, Z.; MOREL, W.; PLOPER, D.; TENUTA, A. Effect of diseases on soybean yield in the top eight producing countries in 2006. Online Plant Health Progress, Saint Paul, 2010.

YANG, X. B.; LUNDEEN, P.; UPHOFF, M. D. Soybean varietal response and yield loss caused by Sclerotinia sclerotiorum. Plant Disease, Saint Paul, v. 83, n. 5, p. 456461, 1999.

ZHAO, X.; HAN, Y.; LI. Y.; LIU, D.; SUN, M.; ZHAO, Y.; LV, C.; LI, D.; YANG, Z.; HUANG, L.; TENG, W.; QIU, L.; ZHENG, H.; LI, W. Loci and candidate gene identification for resistance to Sclerotinia sclerotiorum in soybean (Glycine max L. Merr.) via association and linkage maps. The Plant Journal, Oxford, v. 82, n. 3, p. 245-255, 2015. 